\title{
Evaluación de estrategias para promover el aprendizaje usando TIC: \\ el caso de un curso de Temas de Estadística Multivariante
}

\section{Extracto:}

Actualmente algunos maestros implementan diferentes métodos con el fin de promover una educación ligada a la realidad para proporcionar una formación más eficaz y un aprendizaje significativo. Los métodos activos tienen como objetivo aumentar la motivación y crear escenarios en los que la participación de los estudiantes es el eje para lograr un aprendizaje más significativo. Este trabajo trata sobre la implementación de un proceso de innovación educativa en el curso Temas de Estadística Multivariante que se ofrece

\section{Sumario}

1. Introducción

2. Método de intervención y metodología de evaluación en la licenciatura en Ciencias y Técnicas Estadísticas de la Universidad Veracruzana (México). Se describen las estrategias utilizadas: juegos para la recopilación de datos, diseño y desarrollo de proyectos, y realización de presentaciones individuales y de grupo. Las tecnologías de la información y la comunicación (TIC) que se utilizaron son EMINUS, la plataforma de educación distribuida de la Universidad Veracruzana y la gestión de archivos con Dropbox, además de la comunicación a través de WhatsApp. Se usó el software $\mathrm{R}$ para el análisis estadístico y para la elaboración de presentaciones de resultados en foros académicos. Para explorar las percepciones de los estudiantes se realizaron entrevistas en profundidad y se crearon indicadores para evaluar la satisfacción. Los resultados señalan evidencias positivas, concluyendo que los estudiantes se mostraron satisfechos con la forma en que se diseñó e implementó el curso. Los alumnos también declararon que se sentían capaces de aplicar lo que habían aprendido. Las opiniones indican que a partir de estas estrategias se sintieron preparados para su vida profesional. Por último, se incluyen algunas sugerencias para mejorar el curso en próximas ediciones.

Palabras clave: aprendizaje activo, constructivismo, metacognición, software R Project, tecnologías de la información y la comunicación (TIC).

\footnotetext{
${ }^{1}$ M. M. Ojeda Ramírez, académico de tiempo completo, titular C, de la Facultad de Estadística e Informática de la Universidad Veracruzana (México) y miembro de la Academia Mexicana de Ciencias y del Sistema Nacional de Investigadores.

2 Z. S. Márquez Espinosa, egresada de la Facultad de Estadística e Informática de la Universidad Veracruzana (México).
} 


\section{Evaluation of}

\section{strategies to promote learning using ICT: the case}

\section{Multivariate Statistics}

of a course on Topics of

\section{Abstract:}

Currently some teachers implement different methods in order to promote education linked to reality, to provide more effective training and a meaningful learning. Active methods aim to increase motivation and create scenarios in which student participation is central to achieve a more meaningful learning. This paper reports on the implementation of a process of educational innovation in the course of Topics of Multivariate Statistics offered in the degree in Statistical Sciences and Techniques at the Universidad Veracruzana (Mexico). The strategies used as sets for data collection, design and project development and realization of individual and group presentations are described. Information and communication technologies (ICT) used are: EMINUS, distributed education platform of the Universidad Veracruzana, and managing files with Dropbox, plus communication via WhatsApp. The R software was used for statistical analysis and for making presentations in academic forums. To explore students' perceptions depth interviews were conducted and indicators for evaluating the student satisfaction were defined; the results show positive evidence, concluding that students were satisfied with the way that the course was designed and implemented. They also stated that they feel able to apply what they have learned. The opinions put that using these strategies they were feeling in preparation for their professional life. Finally, some suggestions for improving the course in future editions are included.

Keywords: active learning, constructivism, metacognition, software R Project, information and communication technologies (ICT).

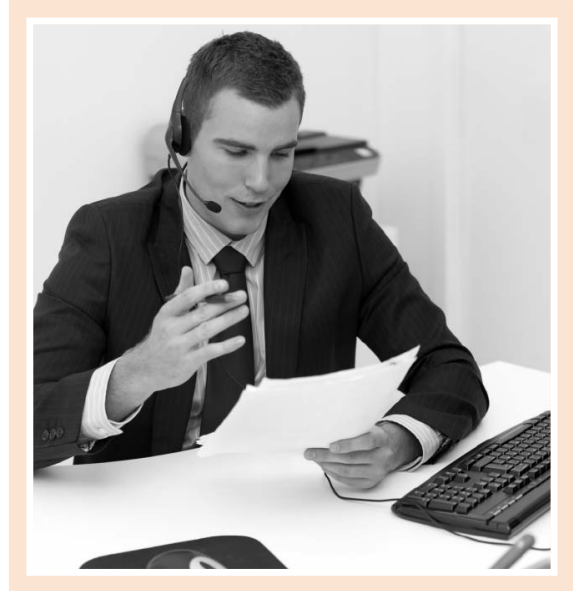

\section{INTRODUCCIÓN}

Las investigaciones que se realizan en las aulas deben tener como finalidad que exista calidad en la educación y que, gracias a ella, se consiga una mejora continua. Por otro lado, el objetivo de usar enfoques innovadores en los cursos es que los estudiantes obtengan aprendizajes significativos y muy vinculados con la realidad de su futura profesión, y que las acciones educativas produzcan efectos motivadores, con la intención de demostrarles que el trabajo organizado y sistemático, el estudio concienzudo de los temas y el uso de herramientas tecnológicas apropiadas repercute en una mejor preparación. En este sentido, la investigación educativa debe servir a los educadores para preparar e impartir mejores cursos con la finalidad de que los estudiantes cada día estén más motivados por los temas de las materias que cursan y que, asimismo, los resultados de estas estrategias innovadoras sean tangibles y evidentes.

Se puede señalar que hoy en día existen estrategias de aprendizaje en las que el profesor implementa procesos cognitivos con pequeños proyectos basados en la realidad; es decir, en problemas reales. Estas estrategias son utilizadas por el docente para que los estudiantes se motiven más y, por ende, se desenvuelvan mejor en las clases. 
Estas opciones se encuentran entre una amplia gama denominada "estrategias de aprendizaje activo» (DíazBarriga, 2010), que se usan con la finalidad de que los profesores puedan generar procesos para la construcción de conocimientos y habilidades de manera conjunta con actitudes de aprecio hacia lo que se estudia; es decir, con ayuda de la teoría, la motivación y la práctica, trabajando con problemas directamente conectados con la realidad de la materia o del tema en cuestión.

El aprendizaje basado en proyectos (ABP) es una de las estrategias que ayuda a los aprendices a plantear, desarrollar y evaluar los proyectos con el objetivo de que puedan resolver problemas del mundo real inmediato. Su finalidad es que los estudiantes desarrollen capacidades, habilidades, que construyan ideas e innoven, todo con ayuda de los conocimientos adquiridos en las aulas y con experiencias socioculturales (constructivismo). Esto es posible gracias a que este enfoque mueve la metacognición, que se encarga de que los alumnos, al involucrarse en un tema, empiecen a recordar aprendizajes o experiencias, y así pongan al servicio del proyecto todo el bagaje de conocimientos que han obtenido. Pero también la motivación se potencia de este modo, por lo que los estudiantes se involucran para trabajar con mayor empeño y compromiso (Garrigós y Valero-García, 2012).

Hoy en día la tecnología ha invadido todas las esferas de nuestra vida. Es imposible obviar que las TIC nos brindan maravillosas oportunidades para mejorar los procesos educativos. Gracias a ellas, tenemos mejores comunicaciones y más facilidades para realizar tareas que antes eran impensables. Pero las TIC, así como son una gran ventaja, también nos plantean un reto. En el contexto de un curso concreto, tanto el profesor como los estudiantes deben aprender a usarlas y a explotar todo el potencial que ofrecen para hacer más eficientes los procesos de aprendizaje. Gracias a las TIC se puede obtener un software libre llamado «R Project» (Culpepper y Aguinis, 2011), con el que los estudiantes pueden realizar cualquier análisis estadístico. Un estudio relevante que muestra cómo se implementa el uso de este software fue el que se realizó en la Universidad Pública de Navarra, Campus de Arrosadía (Pamplona) (Ugarte, Goicoa y Militino, 2008), donde los estudiantes de Ingeniería que cursaban la materia de Estadística utilizaron este software para manejar mejor el tratamiento de la información, llegando a concluir que los estudiantes aprendían mejor en la práctica.

\section{Los métodos activos tienen como objetivo aumentar la motivación y crear escenarios en los que la participación de los estudiantes es el eje para lograr un aprendizaje más significativo}

Los juegos y los proyectos son una parte esencial para aplicar estrategias de aprendizaje activo, ya que en un estudio realizado en la Universidad de la Laguna, en el Departamento de Ingeniería Informática (González, 2014), se diseñó el aprendizaje basado en juegos y proyectos. Los estudiantes aprendieron de forma dinámica, ya que se sirvieron de estos medios para potenciar el pensamiento creativo e innovador, resolviendo problemas de forma creativa. Se dijo que con los juegos se adquiría la habilidad de comprender cómo y con qué resolver los problemas. Todo esto se implementó con ayuda de las TIC y la plataforma de aprendizaje distribuido de la misma universidad.

Como consecuencia de todo lo expuesto hasta ahora, la licenciatura de Ciencias y Técnicas Estadísticas de la Universidad Veracruzana, programa que se ofrece en la Facultad de Estadística e Informática, situada en la ciudad de Xalapa [Veracruz (México)], trata de innovar cada día, de mantener los cursos actualizados y de brindar aprendizajes significativos a los futuros profesionales de la estadística aplicada. En este sentido, los contenidos que se imparten en la experiencia educativa Temas de Estadística Multivariante han sido estructurados con estrategias de aprendizaje activo para que los estudiantes apliquen lo aprendido con ayuda de las TIC, ya que en los últimos años el sistema de educación pide una actualización en prácticas y teorías para que los alumnos se desarrollen en la sociedad de la información y el conocimiento (UNESCO, 2013).

En este trabajo se describe la implementación de un proceso de innovación educativa en el curso mencionado, presentando las estrategias utilizadas, como juegos para la recopilación de datos, el diseño y el desarrollo de proyectos y la realización de presentaciones individuales y de grupo. 
Las TIC que se utilizaron para el soporte en línea son EMINUS (Colunga y Jiménez, 2007), la plataforma de educación distribuida de la Universidad Veracruzana, y la gestión de archivos con Dropbox, además de la comunicación a través de WhatsApp. Se usó el software $\mathrm{R}$ para el análisis estadístico y para la elaboración de presentaciones de resultados en foros académicos.

Para explorar las percepciones de los estudiantes se realizaron entrevistas en profundidad a partir de las cuales se definieron indicadores de satisfacción. Los resultados mostraron evidencias positivas, concluyendo que los alumnos estaban satisfechos con la forma en que se diseñó e implementó el curso. Los alumnos también declararon que se sentían capaces de aplicar lo que habían aprendido. Indicaron que a partir de estas estrategias estaban siendo preparados para su vida profesional.

Por último, se incluyeron algunas sugerencias para mejorar el curso en ediciones futuras.
Para explorar las percepciones de los estudiantes se realizaron entrevistas en profundidad a partir de las cuales se definieron indicadores de satisfacción. Los resultados mostraron evidencias positivas, concluyendo que los alumnos estaban satisfechos con la forma en que se diseñó e implementó el curso

\section{MÉTODO DE INTERVENCIÓN Y METODOLOGÍA DE EVA- LUACIÓN}

En la Universidad Veracruzana, en su sede de Xalapa, se encuentra la Facultad de Estadística e Informática, que ofrece la licenciatura en Ciencias y Técnicas Estadísticas. En esta carrera se imparte la experiencia educativa llamada Temas de Estadística Multivariante, que se localiza en el Área de Formación Terminal, considerada dentro de las materias optativas. Es una de las asignaturas avanzadas del plan de estudios y resulta importante cursarla porque en ella se utilizan técnicas para analizar inferencialmente conjuntos de datos multivariantes. Esta materia tiene un alto contenido teórico, por lo que es imprescindible que el aprendizaje teórico se ponga en práctica con situaciones reales. La idea al impartir la clase es que los estudiantes obtengan una enseñanza-aprendizaje con estrategias activas; es decir, que desarrollen proyectos con datos reales. Los temas que se cubren incluyen la inferencia básica multivariante bajo el supuesto de normalidad, la comparación de varias poblaciones y el análisis discriminante. En esta asignatura se utilizaron juegos para recoger datos, como la rayuela univariante y bivariante; se recolectaron datos de mediciones de frutos de tres especies diferentes, con los que se midió la altura, el ancho y el peso de los frutos; se diseñaron y desarrollaron proyectos, en los que se definieron los objetivos inferenciales multivariantes que se asociaron a preguntas de investigación, considerando los temas establecidos en el programa de la materia. Las sesiones se programaron con revisiones teóricas de los temas, demostraciones prácticas de cómo implementar las técnicas usando el software libre R Project, y durante la solución de problemas se realizaron exposiciones, tanto del profesor -para establecer las pautas-, como de los estudiantes, en las que el profesor fomentó la participación grupal haciendo retroalimentaciones. El curso utilizó intensivamente las TIC para que en cualquier momento hubiese comunicación entre el profesor y los estudiantes, haciendo uso, para ello, de la plataforma de la universidad, llamada EMINUS. También se utilizó Dropbox, para gestionar el diseño y el desarrollo de los proyectos, así como WhatsApp y el correo electrónico, para mantener una comunicación eficiente. Los proyectos, una vez terminados y mejorados en varias ocasiones, se presenta- 
ron en el V Foro de Estadística Aplicada, realizado en Xalapa en mayo de 2015. Se montaron tres carteles y se realizó una ponencia oral. Esto último con la finalidad de que los estudiantes fueran adquiriendo experiencia en este tipo de eventos académicos.

Los alumnos inscritos en este curso entre febrero y agosto de 2015 fueron 8 . A estos estudiantes se les aplicó una entrevista en profundidad semiestructurada que constaba de 10 preguntas. La finalidad de este cuestionario no fue otra que la de conocer su nivel de agrado con las estrategias de aprendizaje activo para saber si estas resultaron útiles en su aprendizaje y, si no fue así, averiguar el porqué, con la idea de obtener retroalimentaciones para mejorar el curso en ediciones sucesivas. Con las respuestas a las entrevistas se construyeron indicadores de satisfacción usando una escala de agrado: mucho, regular, poco y muy poco; esto dependiendo de un análisis detallado de todos los elementos en la respuesta.

En este trabajo se realizaron análisis basados en estadísticas descriptivas e imágenes de nubes de palabras para sistematizar las opiniones en extenso de los estudiantes respecto a los diferentes aspectos considerados en el curso. Con la finalidad de apreciar a los estudiantes en todas las dimensiones de la innovación aplicada al curso se elaboraron indicadores y, para conocer qué individuos tenían semejanzas y diferencias, se realizó un análisis cluster (Seber, 1984). Con todo esto lo que se buscaba era conseguir elementos con los que poder explicar la tendencia de las percepciones respecto a la experiencia vivida en el curso.

Los análisis descriptivos se realizaron en el paquete estadístico Statistica versión 7 (StatSoft, 2016). Las nubes de palabras se elaboraron en la aplicación gratuita on-line Wordle.net (http://www.wordle.net/). El análisis cluster se realizó usando el software $\mathrm{R}$ Project versión Rx64 2.15.2 (https://www.r-project.org/).

\section{El curso utilizó intensivamente las TIC para que en cualquier momento hubiese comunicación entre el profesor y los estudiantes}

\section{RESULTADOS Y DISCUSIÓN}

En el curso se inscribieron 8 estudiantes (4 hombres y 4 mujeres), con edades comprendidas entre los 21 y los 37 años: 7 de ellos tenían entre 21 y 23 años; 1 tenía 37 años; el $75 \%$ cursaban el último año de su proceso formativo.

En la figura 1 se presenta una gráfica de barras múltiples que muestra la opinión sobre las estrategias participativas que se implementaron en el curso. Se observa que el $75 \%$ de los participantes consideraron las exposiciones del profesor de mucha utilidad, mientras que el $25 \%$ restante las valoró como regular. En las exposiciones de los alumnos, el $50 \%$ dijo que resultaron de mucha importancia, el 12,5\% las consideró regular y el $37,5 \%$ afirmó que estas exposiciones no fueron de relevancia para el aprendizaje; es decir, que las exposiciones de sus compañeros sí eran importantes, pero no las veían de gran utilidad para aprender la ma-

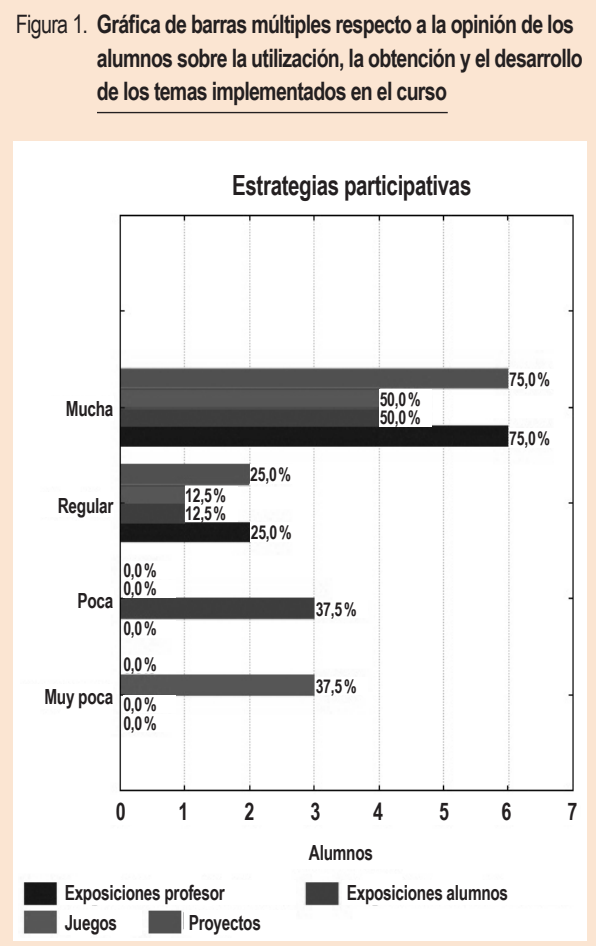

Fuente: elaboración propia 
teria. En lo que respecta a los juegos, el $50 \%$ los consideraron útiles, el $12,5 \%$ dijo que regular y el $37,5 \%$ expuso que fueron de muy poca utilidad; es decir, que a la mitad de los estudiantes sí les gustó aprender con juegos, pero la otra mitad valoró que esta estrategia no era muy relevante. En relación a los proyectos, un $75 \%$ de los estudiantes pensó que eran de mucha importancia y el $25 \%$ los declaró como de regular importancia. Para concluir, podemos decir que la mayoría de los estudiantes consideraron de gran utilidad las exposiciones del profesor y los proyectos que realizaron, pero expresaron que eran de poca o de muy poca utilidad tanto los juegos como las exposiciones de sus compañeros.

En la figura 2 se observa la importancia que los alumnos le dieron a los temas del curso. En relación al tema de Distribución Normal Multivariante, el 37,5\% lo consideró muy importante, el $50 \%$ lo catalogó como importante, y el $12,5 \%$, como moderadamente importante; es decir, la mayoría valoró este tema como importante.
Para el tema de la $\mathrm{T}^{2}$ de Hotelling, el $25 \%$ dijo que era importante, el $62,5 \%$, moderadamente importante, y el $12,5 \%$ lo consideró poco importante; es decir, la mayoría de los estudiantes expresaron que era importante, pero no tanto como otros temas. En el tema de Intervalos de Confianza, el $25 \%$ indicó que era muy importante, el $12,5 \%$, que era moderadamente importante, y el $65,5 \%$ opinó que era poco importante; es decir, la mayoría valoró este tema como de muy poca importancia. Para el tema de Análisis de Varianza Multivariante, el $37,5 \%$ indicó que era muy importante, el $25 \%$, importante, el $12,5 \%$, moderadamente importante, y el $25 \%$, poco importante; es decir, que la mayoría opinó que el tema era gran de importancia. En conclusión, podemos decir que la mayoría de los alumnos indicaron que eran de poca importancia y moderadamente importantes los temas de $\mathrm{T}^{2}$ de Hotelling y de Intervalos de Confianza; los temas que los alumnos consideraron muy importantes fueron la Distribución Normal Multivariante y el Análisis de Varianza Multivariante.

\section{Figura 2. Gráfica de barras múltiples sobre la importancia que los estudiantes le dieron a los temas impartidos}

Temas del curso que los estudiantes consideran más importantes

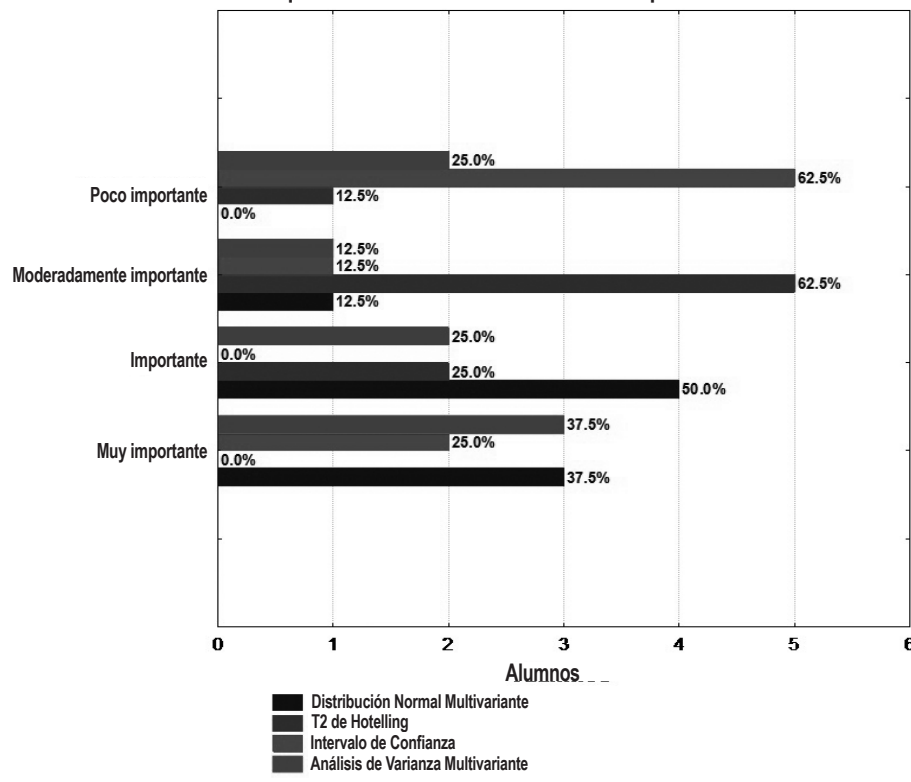

Fuente: elaboración propia. 
Figura 3. Gráfica de barras múltiples respecto a la utilidad de las TIC que se utilizaron en el curso Temas de Estadística Multivariante

Grado de utilidad que los estudiantes le dieron a las TIC implementadas en el curso

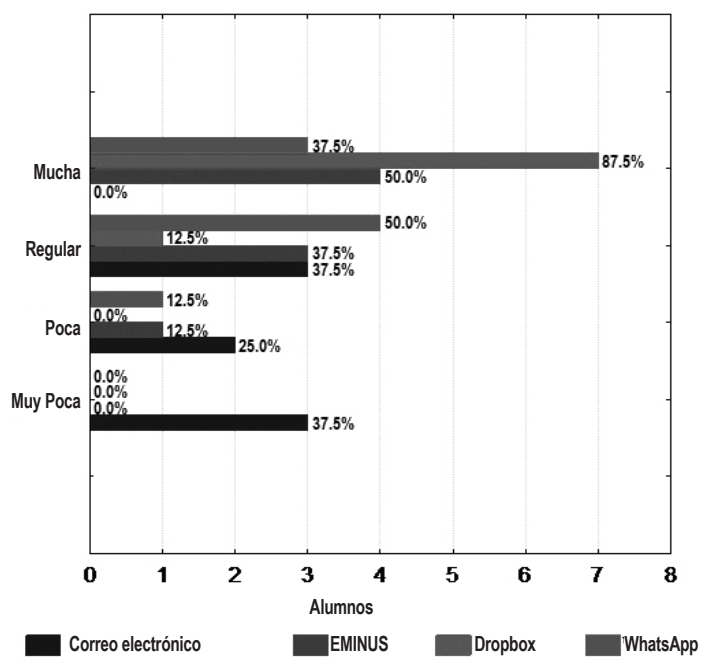

Fuente: elaboración propia

En la figura 3 se observa que el $37,5 \%$ de los participantes pensaba que el Correo Electrónico era de muy poca utilidad, el $25 \%$, que era poco importante, y el $37,5 \%$ lo consideraba como regular; es decir, que algunos estudiantes lo veían de poca utilidad, otros, no tenían inconveniente si lo utilizaban o no, y el resto que era poca la utilidad. En relación a la plataforma EMINUS, el $12,5 \%$ lo consideraba de muy poca utilidad, el $37,5 \%$, regular, y el $50 \%$ indicaba que era de mucha importancia; es decir, que la mayoría de los estudiantes pensaba que la plataforma era de mucha utilidad para la clase. Respecto al uso de Dropbox, el 12,5\% le daba una puntuación regular y el $87,5 \%$ indicaba que era de mucha utilidad. Para WhatsApp, el 12,5\% consideró que era de poca utilidad, el $50 \%$, regular, y el $37,5 \%$, de mucha utilidad; es decir, la mayoría de los alumnos no tenían inconveniente si utilizaban o no el WhatsApp. En conclusión, los estudiantes preferían utilizar Dropbox para enviar y recibir archivos y catalogaron el correo electrónico como de muy poco importante.

Como se puede observar en la figura 4, todos los alumnos consideraron que trabajar con el software libre $R$ era importante para el análisis estadístico. Dicho software libre $\mathrm{R}$, herramienta que se implementó en el curso, es un programa estadístico que sirve para hacer análisis; tiene un lenguaje para utilizarlo; hay que programar para rea-

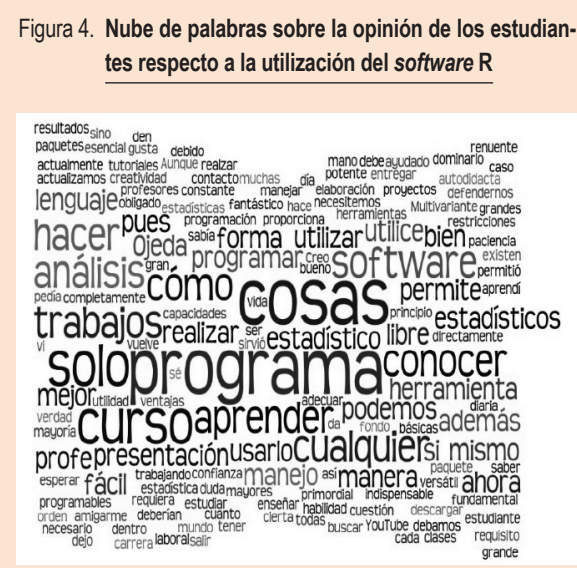

Fuente: elaboración propia 


\section{Los estudiantes opinaron que lo que más les gustó del curso fue el dinamismo del mismo, es decir, trabajar en equipos}

lizar trabajos; es fácil de usar; potencia la imaginación; arroja resultados y gráficos estadísticos de forma adecuada; realiza actividades y trabajos para los temas. Los estudiantes comentaron que al principio les resultó complicado utilizar el paquete, pero después lo entendieron $y$, actualmente, se sienten con mayor capacidad de realizar más trabajos. Piensan que es un software necesario para un estadístico y que les permite desarrollar habilidades. Con ayuda de artículos y libros que les proporcionó el profesor, así como consultando en internet, los alumnos se convirtieron en autodidactas de este paquete.

En la experiencia educativa se cumplió con los temas implementados en el programa. Los estudiantes opinaron que los temas impartidos durante el curso fueron conforme a proyectos donde el profesor explicaba y ponía ejemplos. El profesor les enseñó a recolectar datos reales con tres especies diferentes de frutas, midiendo el ancho, el largo y el peso. Para realizar técnicas mul-

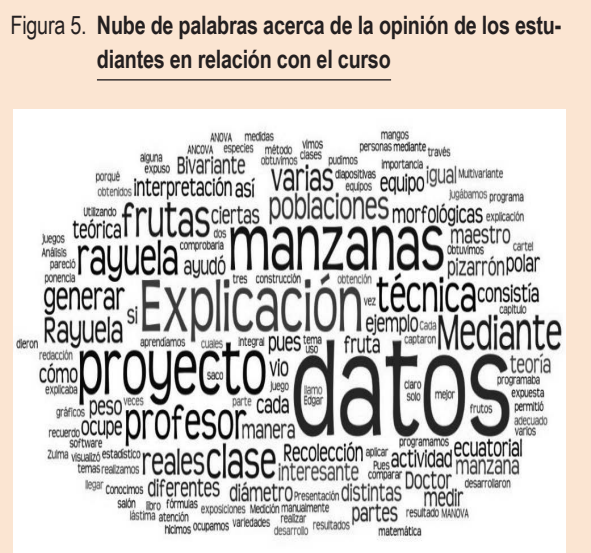

Fuente: elaboración propia. tivariantes, también recolectaron datos por medio de la rayuela, que fue un juego que realizaron en clase. Les resultó interesante realizar la recogida de datos, ya que analizaron e interpretaron los datos exponiendo en clase los resultados. Utilizaron Análisis de Varianza Multivariante, Distribución Normal Multivariante, Elipses de Confianza y $\mathrm{T}^{2}$ de Hotelling, y con ello realizaron una ponencia y tres carteles (véase figura 5).

Durante el curso, en la Facultad de Estadística e Informática tuvo lugar el V Foro de Estadística Aplicada a la Economía, Geografía e Informática. Los estudiantes participaron con los proyectos realizados en clase. En la figura 6 podemos ver una nube de palabras en la que se observan las opiniones de los estudiantes en relación con esta participación. Los alumnos expresaron que participaron por primera vez realizando carteles. Lo vieron como una oportunidad, ya que tuvieron nuevas experiencias, agradables y positivas. También comentaron que lo pudieron hacer gracias al apoyo del profesor. Al principio les preocupaba participar porque les daba miedo presentar el cartel y, además, algunos tenían que realizar una exposición oral. Esta experiencia la vieron como algo positivo y que les permitiría participar en otra experiencia similar más adelante. Otro aspecto positivo de su participación la encontraron en que, al ser algo formal, tuvieron que investigar más, y en que recibieron retroalimentación de otras personas para sus proyectos. Esto les gustó mucho.

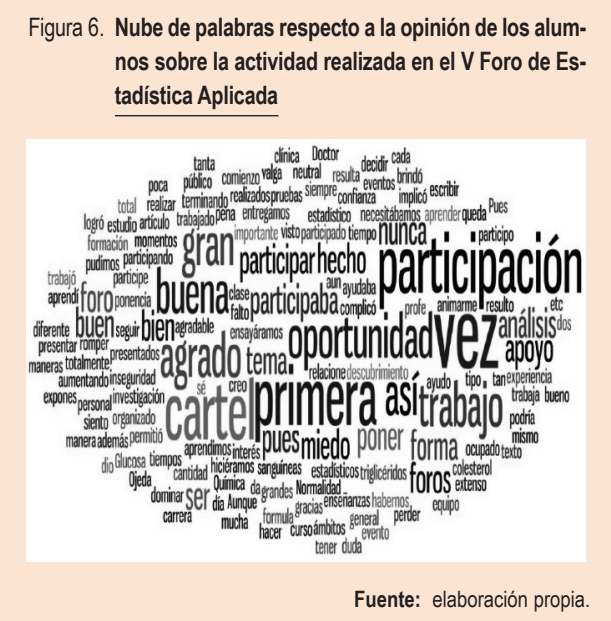


Los estudiantes opinaron que lo que más les gustó del curso fue el dinamismo del mismo, es decir, trabajar en equipos (véase figura 7). El curso fue muy positivo, porque siempre hubo transmisión de conocimientos; adquirieron nuevas experiencias, como participar en el V Foro de Estadística Aplicada, ya fuera presentando los carteles o realizando una ponencia; elaboraron trabajos de manera participativa; les agradó el ambiente de la clase; aplicaron análisis estadísticos en el software R; hubo comunicación con el grupo y con el profesor; cualquier duda que tuvieron la pudieron preguntar y resolver; desarrollaron otras habilidades, como hablar en público; las exposiciones que realizaron fueron mejorando; la clase fue proactiva; etc.

También se les cuestionó sobre si se sentían aptos para aplicar lo aprendido. En la nube de palabras de la figura 8 se puede observar que los alumnos sí se sentían aptos para transmitir los conocimientos adquiridos en clase, desde recoger datos, hasta analizarlos en el software R. Además, participar en el V Foro de Estadística les ayudó a tener más confianza. Recalcaron que fue importante participar en los proyectos desde

Figura 7. Nube de palabras respecto al gusto de los alumnos por el curso Temas de Estadística Multivariante

jamás cosas arena

abordar ocuparon allá habilidad elaborar termina cuales muchas cosas arena letras largo for foro

retos participando Aprender sobretodo impulsó público placenteras organizada impartidasresulto
literatura motor oportunidad CaCvalor totalmente prónrama presentaban importante expresarse creo ojeda asignaba
presentarse
complejos
aportamos
etclase confianza f
intervalosf barreras equipo trabajo experiencias füroconocimiento determinado iniciativa equipo sino partemiedoforos estadistica trabajar igual ponencias abarcar extensopeleadoscompartir motivaciónreales gustado proactiva guio experiencia reversas abarcar extensopeleadoscompartir motivacionreales prof contarnos gráficos pruebas clases seguracartelesmaestro hipótesis proftor tencativaDoctor explicar contarnos aplicado aprendi actividad gratas apliar dos

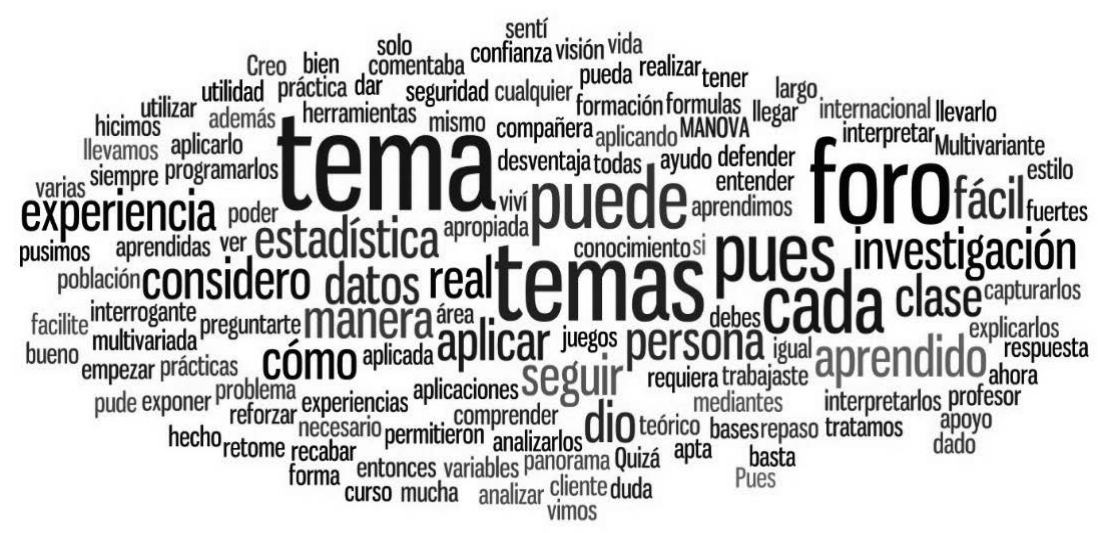

Fuente: elaboración propia 
Figura 9. Nube de palabras respecto a qué piensan los alumnos que se debe mejorar en el curso

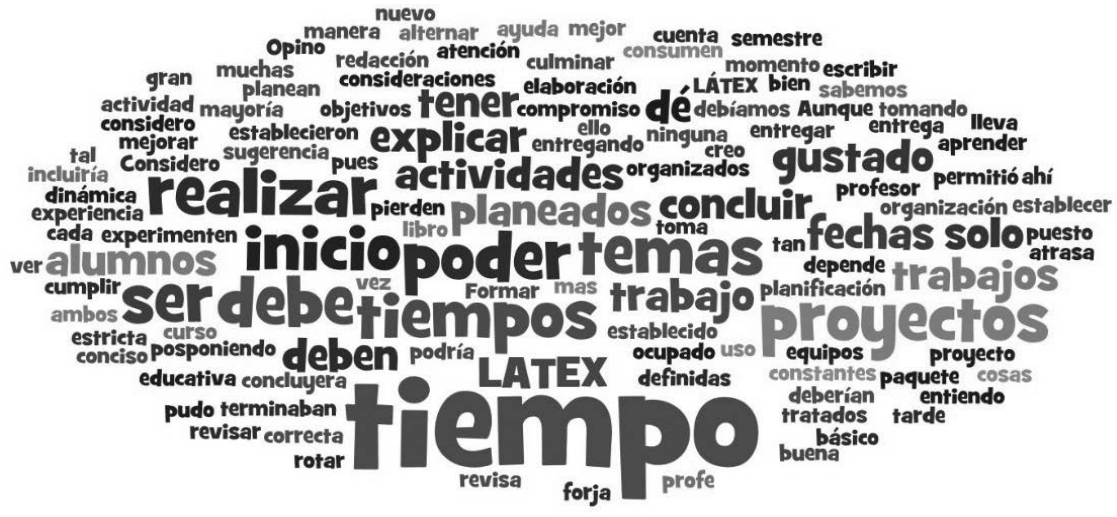

Fuente: elaboración propia.

su inicio, como la recolección de datos, porque esto les hizo encontrarse con problemas reales, algo muy diferente a leer un ejemplo en un libro. Los estudiantes agradecieron el apoyo constante del profesor. Recibieron nuevas experiencias y conocimientos, porque los problemas que se les presentaron con los proyectos hicieron que investigaran más.

Figura 10. Dendrograma respecto a las opiniones de los estudiantes

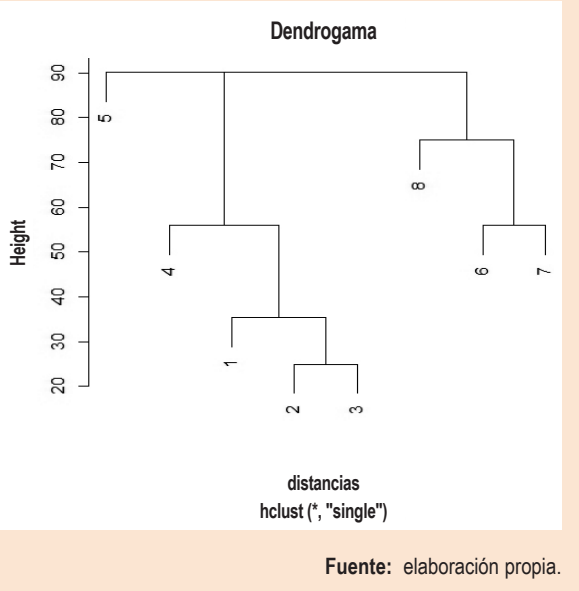

Otro aspecto sobre el que se les preguntó a los alumnos es qué consideraban que se debía mejorar en el curso. En la figura 9 se observa cómo los alumnos piensan que al inicio del curso costó mucho tiempo explicar los proyectos. El semestre pasa muy rápido, de modo que tuvieron que aprender de forma acelerada, lo que llevaba a que las fechas propuestas no se cumplieran. Además, una vez que el profesor mandaba las actividades, luego tardaba mucho tiempo en retroalimentarlas. También, era muy estricto con las fechas de entrega de los trabajos. Finalmente, apuntaron que al inicio del curso se les comentó que verían edición con LATEX, algo que no se llegó a producir.

En la figura 10 se muestra un dendrograma (descripción gráfica de la agrupación según el análisis cluster), donde se presentan dos agrupaciones: a) los estudiantes 1, 2, 3 y 4; b) los estudiantes 6,7 y 8 ; y un estudiante marginal, que es el número 5.

\section{(...) los estudiantes (...) prefirieron Dropbox, por la comodidad de subir y recibir archivos y porque avisa cuando se agregan o realizan modificaciones}


Para concluir, se puede decir que los estudiantes están dispuestos a que los profesores programen proyectos reales porque hacen que estos trabajos reflejen lo aprendido. Además, ante las dificultades existe el apoyo del profesor

\section{CONCLUSIONES}

En este trabajo se obtuvieron resultados de los análisis de la opinión de los estudiantes sobre las estrategias de aprendizaje activo, considerando la utilización de las TIC: la plataforma EMINUS, Dropbox, WhatsApp y correo electrónico, y una estrategia de ABP. Las estrategias de aprendizaje activo son una herramienta fundamental para la práctica. A los estudiantes les agradó desarrollar los proyectos porque analizaron los datos con el software R. Les resultó interesante utilizar este software porque les permitió crear, quitar 0 adicionar gráficas o estructuras para solucionar los problemas. Con los juegos no se sintieron muy cómodos al principio, pero sí con las actividades relacionadas con el proyecto de medir frutas: esta actividad práctica les interesó. Según los estudiantes, la plataforma EMINUS fue de gran utilidad, aunque prefirieron Dropbox, por la comodidad de subir y recibir archivos y porque avisa cuando se agregan o realizan modificaciones. Agradecieron tener una experiencia en el V Foro de Estadística Aplicada porque llevaron a cabo actividades que no habían hecho antes, como elaborar carteles, diseñar una presentación con diapositivas y realizar una ponencia con límite de tiempo. Todo ello les sirvió para mejorar como estudiantes. También opinaron que el semestre fue corto y, por ende, las actividades se tuvieron que entregar rápido, de modo que el profesor tuvo que distribuir el tiempo. Les gustó el curso por la forma en que se implementó.
Los análisis que se realizaron con las preguntas abiertas muestran que la estadística y sus herramientas son tan flexibles que permiten aplicaciones en este tipo de trabajos en los que se tiene información textual. El resto de los análisis se resolvió con las nubes de palabras. La finalidad que tienen estas nubes es dar una visión rápida de un archivo de texto no estructurado: las palabras más frecuentes son las que se ven con mayor claridad porque aparecen en un tipo de letra más grande. Para concluir, se puede decir que los estudiantes están dispuestos a que los profesores programen proyectos reales porque hacen que estos trabajos reflejen lo aprendido. Además, ante las dificultades existe el apoyo del profesor. Asimismo, se demostró que los alumnos apreciaban que se diera importancia a fomentar la habilidad verbal y a realizar presentaciones correctas, ya que estas competencias serán positivas para su desenvolvimiento profesional. Se recomienda una mejor planificación del tiempo; definir con suficiente antelación los proyectos; establecer los lineamientos para las presentaciones; hacer más prácticas con el software $\mathrm{R}$ usando datos reales; que el profesor retroalimente a tiempo. En general, las experiencias con metodologías activas para promover el aprendizaje y la explotación correcta de las TIC disponibles permiten elevar el nivel de satisfacción en los cursos, por muy complejos que sean los contenidos.

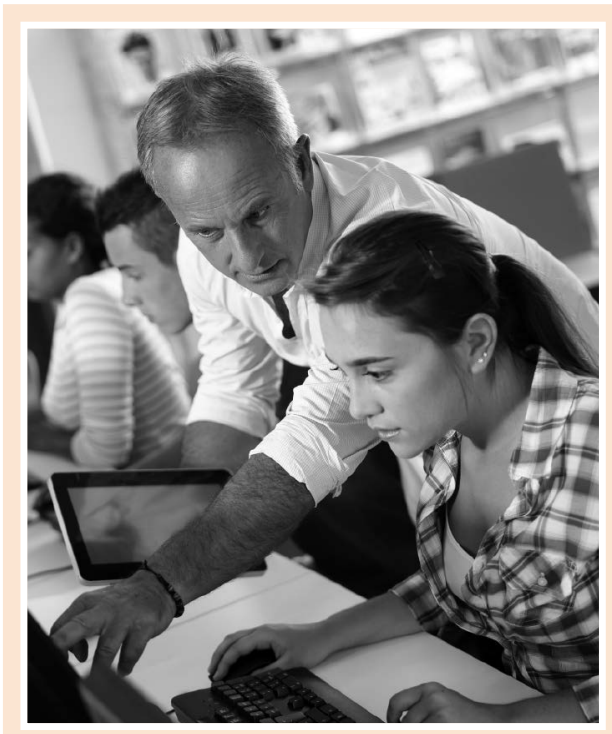




\section{BIBLIOGRAFÍA}

Colunga, J. A. y Jiménez, J. C. [2007]: Eminus. Sistema de Educación Distribuida. La Escuel@ del Futuro, Conferencia presentada en Virtual Educa (Brasil). Disponible en: http://reposital.cuaed.unam. mx:8080/jspui/bitstream/123456789/1169/1/16 JCM.PDF [Consultado: 4 de julio de 2016].

Culpepper, S. A. y Aguinis, H. [2011]: «R is for revolution: a cutting-edge, free, open source statistical package», Organizational Research Methods, 14 (4), págs. 735-740.

Díaz-Barriga, F. [2010]: «Los profesores ante las innovaciones curriculares», Revista Iberoamericana de Educación Superior 1 (1). Disponible en: http://ries. universia.net/index.php/ries/article/view/35/innova [Consultado: 4 de julio de 2016 ].

Garrigós Sabaté, J. y Valero-García M. [octubre-diciembre 2012]: «Hablando sobre aprendizaje basado en proyectos con Júlia», Revista de Docencia Universitaria, 10 (3), págs. 125-151.

González, C. [2014]: «Estrategias para trabajar la creatividad en la educación superior: pensamiento de diseño, aprendizaje basado en juegos y en proyectos», Revista de la Educación a Distancia, 40, págs. 1-15.

R Project [2016]: https://www.r-project.org/ [Consultado: 6 de julio de 2016].

Seber, G. A. F. [1984]: Multivariate observations, Nueva York: Wiley.

StatSoft [2016]: http://www.statsoft.com/Products/STATISTICA/Product-Index [Consultado: 4 de julio de 2016].

Ugarte, D.; Goicoa, T. y Militino, F. [2008]: Pruebas de seguimiento y sesiones de control en la asignatura Métodos Estadísticos de la Ingeniería, Universidad Europea. Disponible en: http://abacus.universidadeuropea.es/handle/11268/3135 [Consultado: 6 de julio de 2016].

UNESCO [2013]: Enfoques estratégicos sobre las TIC en educación en América Latina y el Caribe, Chile: Oficina Regional de Educación para América Latina y el Caribe (OREALC/UNESCO Santiago).

\section{PUBLICIDAD}

\section{Uั
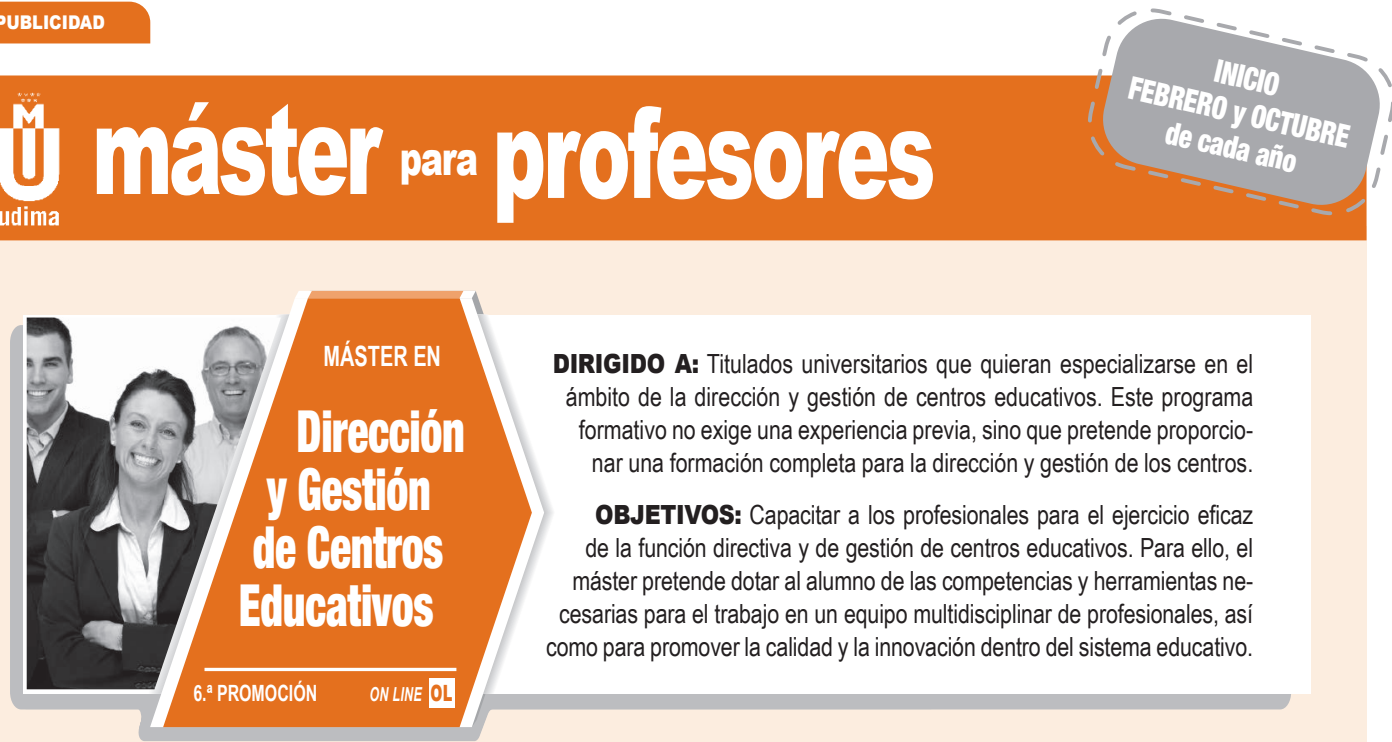

DIRIGIDO A: Titulados universitarios que quieran especializarse en el ámbito de la dirección y gestión de centros educativos. Este programa formativo no exige una experiencia previa, sino que pretende proporcionar una formación completa para la dirección y gestión de los centros.

OBJETIVOS: Capacitar a los profesionales para el ejercicio eficaz de la función directiva y de gestión de centros educativos. Para ello, el máster pretende dotar al alumno de las competencias y herramientas necesarias para el trabajo en un equipo multidisciplinar de profesionales, asi como para promover la calidad y la innovación dentro del sistema educativo. 